\title{
ON THE ARITHMETIC AND HOMOLOGY OF ALGEBRAS OF LINEAR TYPE
}

\author{
BY
}

\author{
J. HERZOG, A. SIMIS AND W. V. VASCONCELOS
}

\begin{abstract}
Three modifications of the symmetric algebra of a module are introduced and their arithmetical and homological properties studied. Emphasis is placed on converting syzygetic properties of the modules into ideal theoretic properties of the algebras, e.g. Cohen-Macaulayness, factoriality. The main tools are certain Fitting ideals of the module and an extension to modules of a complex of not necessarily free modules that we have used in studying blowing-up rings.
\end{abstract}

0. Introduction. The terminology algebras of linear type refers to symmetric algebras of modules and mild modifications thereof. These are broad enough to include various blowing-up rings. To introduce them let $R$ be a commutative Noetherian ring and $E$ a finitely generated $R$-module. Denote by $\operatorname{Sym}(E)$, or simply $S(E)$, the symmetric algebra of $E$ over $R$. We place particular emphasis on the graded structure of $S(E)$ :

$$
S(E)=\bigoplus_{t \geqslant 0} \operatorname{Sym}_{t}(E) .
$$

If $R$ is an integral domain, $S(E)$ is hardly ever an integral domain itself: It is so if and only if each of the symmetric powers $\operatorname{Sym}_{t}(E)$ is a torsion-free $R$-module. It follows easily, however, that if $\operatorname{Sym}_{t}(E)_{0}$ denotes the torsion part of $\operatorname{Sym}_{t}(E)$ then

$$
B(E)=\bigoplus_{t \geqslant 0}\left(\operatorname{Sym}_{t}(E) / \operatorname{Sym}_{t}(E)_{0}\right)
$$

is an integral domain. An important special case is that of $E=I$, an ideal of $R$. $B(I)$ is then the blowing-up ring or, Rees ring

$$
B(I)=\bigoplus_{t \geqslant 0} I^{t}=R[I T], \quad \text { for some indeterminate } T .
$$

The localization of $B(E)$ at $K=R_{(0)}$ yields the polynomial ring

$$
L=K\left[T_{1}, \ldots, T_{e}\right]=\operatorname{Sym}(V),
$$

where $e$ is the rank of $E$, that is, $E \otimes_{R} K=K^{e}=V$.

(3) $C(E)=$ Integral closure of $B(E)$ in $L$.

Note that in the construction of either $B(E)$ or $C(E)$ one may replace $E$ by $E / E_{0}$; we thus assume $E$ is a torsion-free $R$-module.

Received by the editors June 13, 1983.

1980 Mathematics Subject Classification. Primary 13D25, 13F15, 13H10, 14 F05.

Key words and phrases. Approximation complex, blowing-up ring, Cohen-Macaulay ring, $d$-sequence, factorial domain, regular sequence, symmetric algebra.

'Partially supported by NSF grant MCS-8301870.

(C)1984 American Mathematical Society $0002-9947 / 84 \$ 1.00+\$ .25$ per page 
For the next algebra suppose $R$ is also integrally closed. In this case, for each prime ideal $P$ of height 1 of $R, E_{P}$ is a free $R_{P}$-module and thus $\operatorname{Sym}_{t}\left(E_{P}\right)=$ $\left(\operatorname{Sym}_{t}(E)_{P}\right)$ embeds in $\operatorname{Sym}_{t}(V)$; it then follows that $\operatorname{Sym}_{t}(E)^{* *}(=$ $\left.\operatorname{Hom}_{R}\left(\operatorname{Hom}_{R}\left(\operatorname{Sym}_{t}(E), R\right), R\right)\right)$, the $R$-bidual of $\operatorname{Sym}_{t}(E)$, is a submodule of $\operatorname{Sym}_{t}(V)$. In particular,

$$
D(E)=\bigoplus_{t \geqslant 0} \operatorname{Sym}_{t}(E)^{* *} \quad(=\text { graded bidual of } S(E))
$$

is a subring of $L$. It turns out that $C(E)$ itself is a subring of $D(E)$.

Each of the algebras $B(E), C(E)$ and $D(E)$ displays properties that would be of interest to have in $S(E)$. Thus $B(E)$ is an integral domain, while $C(E)$ and $D(E)$ are Krull domains. Furthermore, $D(E)$ is always factorial along with $R$. On the other hand, even for geometric rings, $D(E)$ may show some pathology: e.g. $D(E)$ may not be Noetherian (cf. Example 2.3), although no such example is known for $R$ regular.

We now outline the contents, leaving further comments to the appropriate sections. We study some arithmetical properties of these four algebras and consider comparisons in the sequence of homomorphisms ( $R=$ normal): $S(E) \rightarrow B(E) \rightarrow$ $C(E) \rightarrow D(E)$. To this purpose we attempt several approaches at converting syzygetic properties of the module $E$ - the fine details of a projective resolution of $E$ -into ideal theoretic properties of $S(E)$. When $R$ is a Cohen-Macaulay domain, a first level of necessary conditions for various equalities in the sequence above is obtained by considering the heights of the Fitting ideals of a presentation

$$
R^{m} \stackrel{\phi}{\rightarrow} R^{n} \rightarrow E \rightarrow 0
$$

A recurring requirement involves sliding estimates on the sizes of the ideals $I_{t}(\phi)$ generated by the $t$-sized minors of $\phi$ of the type

$\left(\mathscr{F}_{k}\right) \quad \operatorname{height}\left(I_{t}(\phi)\right) \geqslant \operatorname{rank}(\phi)-t+1+k, \quad 1 \leqslant t \leqslant \operatorname{rank}(\phi)$.

Thus the equality $S(E)=B(E)$ requires $\left(\mathscr{F}_{1}\right)$, while $S(E)=D(E)$ needs $\left(\mathscr{F}_{2}\right)$. None of these conditions is, in general, sufficient.

Full comparisons between any two such algebras present varying degrees of difficulty. Surprisingly, the equality $C(E)=D(E)$, for $R=$ Cohen-Macaulay, can be essentially decided at the level of the ideals $I_{t}(\phi)$ (Theorem 2.1). Testing for the equality $S(E)=C(E)$ will be largely ignored here and we shall focus on the equalities $S(E)=B(E)$ and $S(E)=D(E)$.

After discussing some of the arithmetical properties of these algebras in $\$ \S 1$ and 2 , we introduce the $Z$-complex, $Z(E)$, of the module $E$. It is but a simple extension of one of the so-called approximation complexes of [12-14], and used there to study Rees rings and associated graded algebras.

The construction hinges on the Koszul homology modules $H_{i}\left(S_{+} ; S(E)\right)\left(S_{+}=\right.$ irrelevant ideal of $S(E)$ ). The $i$ th graded component of this module, $Z_{i}=$ $H_{i}\left(S_{+} ; S(E)\right)_{i}$, is often accessible from other properties of $E$. (For instance, when $E$ is an ideal $I$, the $Z_{i}$ are the modules of cycles of an ordinary Koszul complex on $I$.) 
These modules can be put together into a complex of graded $\tilde{S}=S\left(R^{n}\right)$-modules:

$$
Z(E): 0 \rightarrow Z_{l} \otimes \tilde{S}[-l] \rightarrow Z_{l-1} \otimes \tilde{S}[-l+1] \rightarrow \cdots \rightarrow Z_{1} \otimes \tilde{S}[-1] \rightarrow \tilde{S} \rightarrow 0
$$

$(l=n$-rank $(E))$. Since $H_{0}(Z(E))=S(E)$, and the complex is relatively short, it often turns that properties of $S(E)$ can be read off $Z(E)$.

To indicate the range of applicability and flexibility of the $Z$-complex, in $\$ 4$ we discuss broad classes of examples where it is possible to transfer properties from $E$ to $S(E)$. Since the case of ideals has been dealt with elsewhere $[12,13,14,29$ and 30], the emphasis is now on modules of higher rank giving rise to integral domains or Cohen-Macaulay algebras.

The sequential criterion of acyclicity for the case of ideals of [13] is extended in $\$ 5$ to torsion-free modules. As a consequence one obtains the following description of modules $E$ for which $Z(E)$ is acyclic (Theorem 5.6): For $R=$ normal, $E$ must arise from a (Bourbaki-) sequence $0 \rightarrow F \rightarrow E \rightarrow I \rightarrow 0$, where $I$ is an ideal generated by a proper sequence (the known condition for acyclicity of $Z(I)$ ) and $F$ is a free module and its basis forms a regular sequence on $S(E)$.

An important technical device is obtained by studying a resolution of $S(E)$ as an $\tilde{S}=S\left(R^{n}\right)$-module whenever $Z(E)$ is acyclic. It allows us, in $\S 6$, to derive very precise information on $E$ if $S(E)$ is to be Cohen-Macaulay and the full determination of the CM type of $S(E)$.

The last section deals with a discussion of a conjecture to the effect that if $S(E)$ is factorial, then $E$ must have projective dimension at most 1 ( $R=$ regular). There is a considerable body of evidence for it and connections to the existence of modules with rather 'strange' properties. These resemble some of the difficulties of building indecomposable vector bundles over $P_{n}$, for $n$ large.

The rings considered in this paper will be commutative Noetherian (except for one instance) with an identity. For notation, terminology and basic results-especially when dealing with Cohen-Macaulay rings - we shall use [18 and 22].

1. Samuel's criterion. In [28] Samuel proved some elementary but ultimately very interesting properties of graded factorial rings. It exploits the relationship between factoriality of a ring $A=\oplus_{t \geqslant 0} A_{t}$ and the $A_{0}$-module structure of the components $A_{t}$ typified in the following:

Theorem 1.1. Let $A=\oplus A_{t}$ be a factorial graded Noetherian domain. Then $A_{0}$ is factorial and each $A_{t}$ is a reflexive $A_{0}$-module.

More generally, if $A$ is integrally closed (= normal) the condition that each $A_{t}$ be $A_{0}$-reflexive is equivalent to the condition that divisorial primes of $A$ (i.e. height 1 primes) contract to 0 or divisorial primes of $A_{0}$. This is usually denoted PDE, cf. [9], where it is discussed in detail.

When applied to $S(E)$ this gives the more precise $[9,28]$ :

THEOREM 1.2. Let $R$ be a normal Noetherian domain and let $E$ be a finitely generated $R$-module. Suppose each $\operatorname{Sym}_{t}(E)$ is a reflexive $R$-module. Then $S(E)$ is a Krull domain and $\mathrm{Cl}(R)=\mathrm{Cl}(S(E))(\mathrm{Cl}(-)$ denotes the divisor class group.). 
Referring to the algebras $C(E)$ and $D(E)$ of $\S 0$ :

Proposition 1.3. Let $R$ be a normal Noetherian domain and a finitely generated $R$-module. Then $C(E)$ and $D(E)$ are (graded) Krull domains.

Proof. For $C(E)$ it is clear since $B(E)$ is a Noetherian domain. As for $D(E)$, we shall define a family of divisorial prime ideals with the requisite finite character of a Krull domain. First, however, we recall [9]:

Lemma 1.4. Let $R$ be a normal Noetherian domain and $M$ a finitely generated torsion-free $R$-module. The following are equivalent:

(a) $M$ is reflexive.

(b) $M=-M_{p}$ over the height 1 primes of $R$.

(c) Every regular sequence of 2 elements on $R$ is a regular sequence on $M$.

To complete the proof of 1.3 , consider the following (prime) ideals of $D(E)$ :

(i) $P=Q \cap D(E)$, where $Q$ is a height 1 prime of $L$.

(ii) $P=\oplus_{t \geqslant 0}\left(P_{0} \operatorname{Sym}_{t}(E)\right)^{* *}$, where $P_{0}$ is a height 1 prime of $R$.

It is clear that both (i) and (ii) are divisorial primes of $D(E)$ and that $D(E)_{P}=$ discrete valuation domain. Furthermore, using 1.4, it follows that the prime ideals obtained have the finite character property of a Krull domain [18].

In the special case that $R$ is, in addition, factorial, one has, from Nagata's lemma [22]:

COROllary 1.5. Let $R$ be a factorial Noetherian domain and $E$ a finitely generated $R$-module. Then $D(E)$ is a factorial domain.

2. Finiteness. We now compare the algebras $C(E)$ and $D(E)$ in terms of a free presentation of the module $E$. Let $R$ be a normal Noetherian domain and let $E$ be a module with a presentation $R^{m} \stackrel{\phi}{\rightarrow} R^{n} \rightarrow E \rightarrow 0$. Denote by $I_{t}(\phi)$ the ideal generated by the $t$-sized minors of a matrix representation $\left(a_{i j}\right), 1 \leqslant i \leqslant m, 1 \leqslant j \leqslant n$, of $\phi$. Although the $I_{t}(\phi)$ are not true invariants of $E$, note that the Fitting invariants $F_{s}(E)$ are just $F_{s}(E)=I_{n-s}(\phi)$. It is convenient, however, to work from a fixed presentation of $E$.

We consider the following condition on the ideals $I_{t}(\phi)$. Let $k \geqslant 0$ be an integer.

$$
\operatorname{ht}\left(I_{t}(\phi)\right) \geqslant \operatorname{rank}(\phi)-t+1+k, \quad 1 \leqslant t \leqslant \operatorname{rank}(\phi) .
$$

In terms of the Fitting ideals this can be written

$$
\operatorname{ht}\left(F_{s}(E)\right) \geqslant s-\operatorname{rank}(E)+1+k, \quad \operatorname{rank}(E) \leqslant s .
$$

In turn, these global conditions can - by an immediate localization argument-be expressed in terms of the local number of generators of the module $E$ :

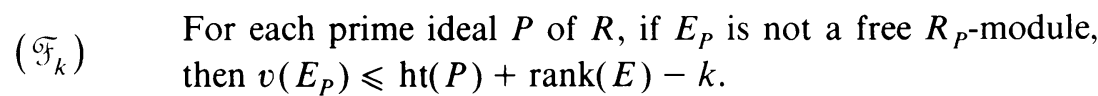

$(v(-)=$ minimum number of generators of $(-)$.)

REMARKs. (i) These properties could also be defined for a nondomain, at least as long as the module $E$ admits a rank. This condition is equivalent to saying that the ideal generated by the largest sized minors contains regular elements. 
(ii) When $R$ is a Cohen-Macaulay ring these conditions are important in the study of the Krull dimension of $S(E)$ (see also [30]). Thus, for instance, $\left(\mathscr{F}_{0}\right)$ means that, locally, $\operatorname{dim} S(E)=\operatorname{dim} R+\operatorname{rank}(E) .\left(\mathscr{F}_{k}\right), k \geqslant 1$, has the following interpretation. In the definition above, consider the case $t=\operatorname{rank}(\phi)$ so that grade $I_{t}(\phi) \geqslant k$ +1 . Let $P$ be a prime of $R$ and let $\mathbf{x}=\left\{x_{1}, \ldots, x_{l}\right\}, l \leqslant k$, be a regular sequence contained in $P$. Now reduce $E$ modulo $(\mathbf{x}), E^{\prime}=E \otimes(R /(\mathbf{x})), \phi^{\prime}=\phi \otimes(R /(\mathbf{x}))$. Since $I_{t}\left(\phi^{\prime}\right)$ contains regular elements, $\operatorname{rank}(E)=\operatorname{rank}\left(E^{\prime}\right)$, and from the defintion of $\left(\mathscr{F}_{k}\right)$ we see that $E^{\prime}$ satisfies $\left(\mathscr{F}_{k-l}\right)$. Therefore $\operatorname{dim} S\left(E^{\prime}\right)=\operatorname{dim} R^{\prime}+\operatorname{rank}\left(E^{\prime}\right)$, thus proving that $(\mathbf{x}) S(E)$ is an ideal of height $l$. This shows that for any prime ideal of $R$,

$$
\operatorname{ht}(P S(E)) \geqslant \inf \{\mathrm{ht}(P), k\} .
$$

It is clear that if, conversely, $\operatorname{dim} S(E)=\operatorname{dim} R+\operatorname{rank}(E)$ and $S(E)$ satisfies this height condition, then $E$ must satisfy $\left(\mathscr{F}_{k}\right)$.

(iii) It follows that if $S(E)$ is an integral domain then $(E)$ satisfies $\left(\mathscr{F}_{1}\right)$, while if each $\operatorname{Sym}_{t}(E)$ is reflexive then $E$ satisfies $\left(\mathscr{F}_{2}\right)$, since each regular sequence $\{a, b\}$ on $R$ is a regular sequence on each $\operatorname{Sym}_{t}(E)$ and thus on $S(E)$. A point that shall be pursued later is that there are 'very few' modules satisfying $\left(\mathscr{F}_{2}\right)$ and therefore very few factorial domains that are symmetric algebras. This is one of the reasons for bringing up the algebra $D(E)$.

Theorem 2.1. Let $R$ be a normal, Cohen-Macaulay, universally Japanese domain.

(a) If E satisfies ( $\left.\mathcal{F}_{2}\right)$, then $C(E)=D(E)$.

(b) Conversely, if $S(E)$ is a domain and $C(E)=D(E)$, then $E$ satisfies $\left(\mathscr{F}_{2}\right)$.

Proof. We may assume $R$ is a local ring. As noted, $\left(\mathscr{F}_{2}\right)$ implies $\operatorname{dim} S(E)=$ $\operatorname{dim} R+\operatorname{rank}(E)$ and thus $\operatorname{dim} S(E)=\operatorname{dim} B(E)=\operatorname{dim} C(E)$.

Let $f$ be a homogeneous element of $D(E)$. The set $I=\{r \in R \mid r f \in C(E)\}$ is an ideal of height at least 2. From Remark (ii) above, $\operatorname{ht}(I S(E)) \geqslant 2$. If $I \neq R$ we shall find this to be impossible.

For simplicity we first argue the case $S(E)=$ domain, that is, $S(E)=B(E)$. Here we have

$$
\operatorname{ht}(I C(E))=\operatorname{ht}(I C(E) \cap B(E)) \geqslant \operatorname{ht}(I B(E)) \geqslant 2,
$$

the equality at the left following from [23, Theorem 34.8]. As $C(E)$ is a Krull domain and $f$ lies in its field of quotients, this is impossible.

If $S(E)$ is not a domain, $B(E)=S(E) / J$, where $J$ is a prime ideal of height 0 . In this case, $\mathrm{ht}((I S(E)+J / J))$ is still at least two, and the argument applies.

For the converse we check $\left(\mathscr{F}_{2}\right)$ in terms of the local number of generators. Let $m$ be the maximal ideal of $R$. We may assume $\mathrm{ht}(\mathfrak{m}) \geqslant 2$ and $E$ is not free; we must show $v(E) \leqslant \mathrm{ht}(\mathrm{m})+\operatorname{rank}(E)-2=\operatorname{dim} S(E)-2$.

Since a regular sequence $\{a, b\}$ on $R$ is regular on $D(E)$, we have ht $(m D(E)) \geqslant 2$. By the result of Nagata, ht $(\mathrm{m} C(E))=\operatorname{ht}(\mathrm{m} C(E) \cap S(E))$. As $\mathrm{m} S(E)$ is a prime ideal of $S(E), \mathfrak{m} S(E) C(E) \cap S(E)=\mathrm{m} S(E)$, so ht $(\mathrm{m} S(E)) \geqslant 2$. Therefore

$$
v(E)=\operatorname{dim} S(E) / \mathfrak{m} S(E)=\operatorname{dim} S(E)-\operatorname{ht}(\mathfrak{m} S(E)) \geqslant \operatorname{dim} S(E)-2,
$$

as desired. 
A simple context for this theorem is that of modules which are free on the punctured spectrum of a local ring. Let $E$ be such a module over a Cohen-Macaulay ring as above (dim $R \geqslant 2)$.

Corollary 2.2. If $v(E) \leqslant \operatorname{dim} R+\operatorname{rank}(E)-2$, then $C(E)=D(E)$.

A construction of a family of modules over regular local rings with this property - thus yielding factorial $C(E)$ 's - can be found in [33]; see also Example 4.4.

EXAMPLE 2.3. For modules of rank 1 the algebras $C(E)$ and $D(E)$ are almost never equal. Let $R$ be a normal domain and let $E=I$ be an ideal of $R$. As remarked, $B(I)=\oplus I^{t}$, the Rees algebra of $I . C(I)=\oplus \bar{I}^{t}$ where $\bar{J}$ denotes the integral closure of the ideal $J$. As for $D(I)$ one has two cases. If $h t(I) \geqslant 2$, then $\left(I^{t}\right)^{* *}=R$ and $D(I)=R[T]$. If $h t(I)=1$, to write $D(I)$ we may assume $I$ is unmixed, say with a primary decomposition $I=P_{1}^{\left(e_{1}\right)} \cap \cdots \cap P_{n}^{\left(e_{n}\right)}$, where $P^{(e)}=e$ th symbolic power of the prime $P$. It follows easily from 1.4 that $\operatorname{Sym}_{t}(I)^{* *}=I^{(t)}$ has a similar decomposition as $I$, with $e_{i}$ replaced by $t e_{i}$.

Note that if $R$ is factorial then $D(I)$ will be isomorphic to $R[T]$ in all cases. For other rings, however, $D(I)$ may even fail to be Noetherian. Indeed, consider the following example. Let $A=C[x, y, z], y^{2} z+y z^{2}=x^{3}-x z^{2}$ and $R=A_{\text {origin }}$; let $P=(x, y)$. It is easy to see that for this ideal, $\operatorname{Sym}_{t}(P)=P^{t} .(0,0,1)$ is, however, a nontorsion point of the elliptic curve [31] and therefore $P^{(t)}$ is nonprincipal for all $t \geqslant 1$. It follows from this that $D(P)=\bigoplus_{t \geqslant 0} P^{(t)}$ is non-Noetherian (cf. [26]).

REMARK. We know, however, of no example of a module $E$ over a regular local ring $R$ for which $D(E)$ is not Noetherian. For instance, let $E$ be a module given by the presentation (cf. [28]):

$$
\phi=\left[\begin{array}{ll}
a & 0 \\
b & a \\
0 & b \\
c & 0 \\
0 & c
\end{array}\right],
$$

$\{a, b, c\}$ a regular sequence in a regular local ring $R . E$ is a reflexive module and $S(E)$ is a domain $\left([\mathbf{1}, \mathbf{1 5}]\right.$; see also [30]) but does not satisfy $\left(\mathscr{F}_{2}\right)$. Samuel pointed out that $\operatorname{Sym}_{2}(E)$ is not reflexive. In fact, no $\operatorname{Sym}_{t}(E), t \geqslant 2$, is reflexive (cf. $\$ 4$ ). We do not know whether $D(E)$ is Noetherian.

3. The $Z$-complex of a module. Let $R$ be a Noetherian ring and $E$ a finitely generated $R$-module. We extend to $E$ the construction of a complex associated to ideals of $R$ (cf. [12, 13, 14, 29 and 30]). Throughout, for convenience, we shall blur the distinction betwwen a complex and the complex augmented by its 0 th homology.

Denote $S=S(E)$ and its irrelevant ideal by $S_{+}$.

Definition 3.1. $Z(E)=M^{*}\left(S_{+} ; S\right)$ is called the approximation complex of $E$, where $M^{*}\left(S_{+} ; S\right)$ is the complex defined in [14] for the ideal $S_{+}$of the ring $S$.

We point out that $M^{*}\left(S_{+} ; S\right)$ is a complex of graded $\tilde{S}$-modules, where $\tilde{S}$ is a polynomial ring $R\left[e_{1}, \ldots, e_{n}\right]$ over $R$ in as many variables as a chosen set of generators of $S_{+}$. In degree $i$,

$$
M_{i}^{*}=H_{i}\left(S_{+} ; S\right)_{i} \otimes \tilde{S}[-i],
$$


where $H_{i}\left(S_{+} ; S\right)_{i}$ denotes the $i$ th graded part of the Koszul homology $H_{i}\left(S_{+} ; S\right)$ of $S$ with respect to a system $\mathbf{x}=\left\{x_{1}, \ldots, x_{n}\right\}$ of linear generators of $S_{+}$, or, what amounts to the same thing, of $E$. Further, we are using the notation for shifting the graded components of a module: $\tilde{S}[-i]_{j}=\tilde{S}_{j-i}$.

Let us briefly indicate how these complexes come about. Let $F=R^{n} \stackrel{\phi}{\rightarrow} E \rightarrow 0$ be a surjection. Consider the graded algebra - a double Koszul complex- $\mathscr{L}=\{\wedge(F)$ $\left.\otimes S(F) \otimes S(E), \partial, \partial^{\prime}\right\}$. In terms of ordinary Koszul complexes,

$$
\{\mathcal{L}, \partial\}=\mathscr{K}(\mathbf{x} ; S(E)) \otimes S(F) \text { and }\left\{\mathcal{L}, \partial^{\prime}\right\}=\mathscr{K}(\mathbf{x} ; S(F)) \otimes S(E) \text {. }
$$

From the commutativity of the differentials $\partial$ and $\partial^{\prime}$, several complexes arise. In particular one obtains a complex $M\left(S_{+} ; S\right)$, with $M_{i}=H_{i}\left(S_{+} ; S\right) \otimes \tilde{S}[-i]$. While $H_{i}\left(S_{+} ; S\right)$ may be cumbersome to deal with, its $i$ th graded part, $H_{i}\left(S_{+} ; S\right)_{i}$, is given simply as

$$
\begin{gathered}
\operatorname{ker}(\stackrel{i}{\wedge} \underset{\stackrel{\partial}{\rightarrow} \stackrel{i-1}{\wedge} F \otimes E),}{ }, \\
\partial\left(a_{1} \wedge \cdots \wedge a_{i}\right)=\sum(-1)^{j}\left(a_{1} \wedge \cdots \wedge \hat{a}_{j} \wedge \cdots \wedge a_{i}\right) \otimes \phi\left(a_{j}\right) .
\end{gathered}
$$

Note that $H_{1}\left(S_{+} ; S\right)_{1}=\operatorname{ker}(\phi)=$ first syzygy module of $E$, which explains the notation $Z(E)$; see also Lemma 3.3.

If we write $H_{i}\left(S_{+} ; S\right)_{i}=Z_{i}(E)=Z_{i}$,

$$
Z(E): 0 \rightarrow Z_{n} \otimes \tilde{S}[-n] \rightarrow \cdots \rightarrow Z_{1} \otimes \tilde{S}[-1] \rightarrow \tilde{S} \rightarrow H_{0}(Z(E))=S(E) \rightarrow 0 .
$$

The differential of $Z(E)$ is that included by $\partial^{\prime}$. An important point is the actual length of this complex; it will be shown later that if $E$ has a $\operatorname{rank}$, say $\operatorname{rank}(E)=e$, then $Z_{i}=0$ for $i>n-e$. Furthermore, its homology is independent of the chosen presentation $\phi$. Note also that in each degree, we have a complex of finitely generated $R$-modules

$$
0 \rightarrow Z_{n} \otimes \tilde{S}_{t-n} \rightarrow \cdots \rightarrow Z_{1} \otimes \tilde{S}_{t-1} \rightarrow \tilde{S}_{t} \rightarrow \operatorname{Sym}_{t}(E) \rightarrow 0 ;
$$

in this form it is convenient for checking acyclicity (cf. $[12,30])$.

The advantage of considering $M^{*}\left(S_{+} ; S\right)$ rather than the full $M\left(S_{+} ; S\right)$ lies in its simplicity since the higher symmetric powers of $E$ do not get directly involved in its construction. Moreover, one has

LEMMA 3.2. The following conditions are equivalent:

(a) $M\left(S_{+} ; S\right)$ is acyclic.

(b) $Z(E)$ is acyclic.

(c) $H_{i}\left(S_{+} ; S\right)_{j}=0$ for $j>i \geqslant 0$.

Furthermore, if the equivalences hold, then $M\left(S_{+} ; S\right)=Z(E)$.

Proof. (a) $\Leftrightarrow$ (c) $\Leftrightarrow$ (b) If $M\left(S_{+} ; S\right)$ is acyclic, then $H_{i}\left(S_{+} ; S\right)_{j}=0$ for $j>i \geqslant 0$ and, conversely, by $[14,(11.9)]$; clearly in such a case $M\left(S_{+} ; S\right)=Z(E)$.

(b) $\Leftrightarrow$ (a), (c) If $Z(E)$ is acyclic, it may be used to compute $H_{i}\left(S_{+} ; S\right)$, since $\operatorname{Tor}_{j}^{\tilde{S}}\left(\tilde{S} /(\mathbf{e}), M_{i}^{*}\right)=0$ for $j>0$. One concludes that $H_{i}\left(S_{+} ; S\right)_{j}=0$ for $j>i \geqslant 0$, and thus the complexes $M\left(S_{+} ; S\right)$ and $Z(E)$ coincide.

We now compare this construction with the approximation complexes associated with ideals, cf. [14] for notations. 
LEMMA 3.3. (a) Let $E=I$ be an ideal; then $Z(E)$ is the $Z$-complex of $I$.

(b) Let $E=I / I^{2}$ be the conormal module of the ideal $I$; then there exists a natural inclusion $H_{i}^{*}(I ; R) \rightarrow H_{i}^{*}\left(S_{+} ; S\left(I / I^{2}\right)\right)$. If the M-complex of $I$ is acyclic, then $M(I ; R)=Z\left(I / I^{2}\right)$.

Proof. (a) is immediate. (b) The elements of $H_{i}^{*}(I ; R)$ considered as elements of $\wedge^{i}(R / I)^{n}$ are obviously in the kernel of $\wedge^{i}(R / I)^{n} \rightarrow \wedge^{i-1}(R / I)^{n} \otimes\left(I / I^{2}\right)$. The rest of the proof proceeds in the same manner as 3.2.

4. Modified Koszul complexes. We now compare the complex $Z(E)$ to the Koszul complex associated to a presentation

$$
0 \rightarrow Z_{1}(E)=L \stackrel{\psi}{\rightarrow} R^{n}=F \rightarrow E \rightarrow 0 .
$$

It will provide a convenient vehicle for exchanging information between the syzygies of $E$ and depth properties of $S(E)$.

The Koszul complex associated to the map $L \stackrel{\psi}{\rightarrow} F$ is $\mathscr{K}(E)=\wedge(L) \otimes S(F)$ with differential

$$
\partial\left(\left(a_{1} \wedge \cdots \wedge a_{r}\right) \otimes w\right)=\sum(-1)^{j}\left(a_{1} \wedge \cdots \wedge \hat{a}_{j} \wedge \cdots \wedge a_{r}\right) \otimes \psi\left(a_{j}\right) \cdot w .
$$

Since $L=Z_{1}(E)$, the skew-commutative structure of $Z(E)$, and the fact that the various differentials are also derivations, gives rise to a chain map $\mathscr{K}(E) \rightarrow Z(E)$ arising out of the maps $\wedge^{r} L \rightarrow Z_{r}(E)$. Let $e$ be the rank of $E$, that is, assume $E \otimes K=K^{e}$, where $K$ is the total ring of fractions of $R$. Since the constructions above localize, it follows that $Z(E)$ is a complex of length $n-e=l=\operatorname{rank}(L)$. $\mathcal{K}(E)$ on the other hand may be much longer since $\wedge^{r}(L)$ may be nonzero for $r>l$. For the purpose of comparison, it is useful to cut down the length of $\mathscr{K}(E)$. This may be done in several ways, most simply by reducing $\mathscr{K}(E)$ modulo its $R$-torsion. We prefer, however, to carry out another modification.

Suppose, for instance, that $E$ is a torsion-free module; in this case each $Z_{r}(E)$ is a second syzygy module and thus reflexive. Replacing $\wedge^{r} L$ by its double dual $\left(\wedge^{r} L\right)^{* *}$, we get a chain map $\mathcal{K}(E)^{* *} \rightarrow Z(E)$.

These modifications are actual identifications in the following cases.

Proposition 4.1. Let $E$ be a finitely generated $R$-module.

(a) If $E$ is a torsion-free module free at the primes $P$ with depth $R_{P} \leqslant 1$, then $\mathcal{K}(E)^{* *} \cong Z(E)$.

(b) Let $R$ be a Cohen-Macaulay ring; if $E$ has a resolution $0 \rightarrow R^{m} \rightarrow R^{n} \rightarrow E \rightarrow 0$ and satisfies $\left(\mathscr{F}_{0}\right)$, then $\mathcal{K}(E) \cong Z(E)$. In this case $Z(E)$ provides a projective $\tilde{S}$-resolution of $S(E)$. Furthermore, if $R$ is an integral domain, then $S(E)$ is a domain if and only if $E$ satisfies $\left(\mathscr{F}_{1}\right)$.

Proof. (a) Here both $\left(\wedge^{r} L\right)^{* *}$ and $Z_{r}(E)$ are reflexive modules which agree in depth $\leqslant 1$ and thus coincide.

(b) Consider the exact sequence $0 \rightarrow \wedge^{r} R^{m} \rightarrow Z_{r}(E) \rightarrow C \rightarrow 0$. To show $C=0$, it is enough to check the height one primes. But the $\left(\mathscr{F}_{0}\right)$-condition now means 
$v\left(E_{P}\right) \leqslant \mathrm{ht}(P)+\operatorname{rank}(E)$, so $E_{P}$ admits a resolution $0 \rightarrow R_{P} \rightarrow R_{P}^{e+1} \rightarrow E_{P} \rightarrow 0$. The assertion follows from the underlying mapping cone construction of both complexes. As for the $\tilde{S}$-projective resolution of $S(E)$, see [ $\mathbf{1}$ or $\mathbf{3 0}$ ].

REMARK. (i) Note that in the above proposition, (b) means that the graded components of $Z(E)$ provide free resolutions of $\operatorname{Sym}_{t}(E)$; the complex $Z(E)$ is, for this case, an aggregate of some of the resolutions of [20 and 34].

(ii) When $I$ is a perfect ideal of height two, (a) and (b) imply that the modules of cycles of an ordinary Koszul complex of $I$ are all free. This in turn is easily seen to be equivalent to saying that the Koszul homology modules $H_{i}(I ; R)$ are CohenMacaulay; see also [2 and 16].

Before we consider other examples, we point out some duality features of these complexes. First we recall a result of [25]; see also [8].

LEMMA 4.2. Let $M$ be finitely generated of finite projective dimension. Assume $M_{\mathfrak{p}}$ is free for each prime with depth $R_{\mathfrak{p}} \leqslant 1$. If $r=\operatorname{rank}(M)$, then $\operatorname{det}(M)=\left(\wedge^{r} M\right)^{* *}$ is an invertible ideal.

We use this in the context of the complex $\mathscr{K}(E)^{* *}$, where $E$ is a torsion-free module and pd $E<\infty$. Thus for the presentation above, $L$ is a reflexive module and $\left(\wedge^{\prime} L\right)^{* *}$ is an invertible ideal $(l=\operatorname{rank}(L))$. Furthermore, for any integer $r<l$, the canonical pairing

$$
\stackrel{r}{\wedge} \stackrel{l-r}{\wedge} \stackrel{\wedge}{\wedge} L \stackrel{l}{\wedge} L \rightarrow(\stackrel{l}{\wedge} L) * *=\operatorname{det}(L)
$$

yields a mapping $\wedge^{r} L \rightarrow \operatorname{Hom}_{R}\left(\wedge^{l-r} L, \operatorname{det}(L)\right)$ which is an isomorphism in depth $\leqslant 1$.

Corollary 4.3. (a) If $l=2$,

$$
L=\operatorname{Hom}_{R}(L, \operatorname{det}(L))=L^{*} \otimes \operatorname{det}(L),
$$

the standard formula for rank 2 bundles.

(b) If $\operatorname{det}(L)=R$ (e.g. $R=$ factorial, or $L$ admits a finite free resolution), for any $r \leqslant l,\left(\wedge^{r} L\right)^{* *}=\left(\wedge^{l-r} L\right)^{*}$, that is, $Z_{r}(E) \cong Z_{l-r}(E)^{*}$.

EXAmple 4.4. Let us consider in some detail some of the modules of [33]. For a regular local ring or, more generally, for a Cohen-Macaulay local ring, it describes a matrix $(\operatorname{dim} R=n) R^{2 n-3} \stackrel{\psi}{\rightarrow} R^{n}$ of rank $n-1$ which splits on the punctured spectrum of $R$. Denote $E=\operatorname{image}(\psi)$. A first remark is that such a module satisfies $\left(\mathscr{F}_{2}\right)$. In [33] it is proved that pd $E \geqslant n-2$. In fact it is the case that pd $E=n-1$. Indeed, otherwise $\operatorname{coker}(\psi)$ would be torsion-free, free on the punctured spectrum of $R$ so it would be isomorphic to a primary ideal generated by $n$ elements that is, to an ideal generated by a system of parameters. In this case the module of relations $E$ would have at least $\left(\begin{array}{l}n \\ 2\end{array}\right)$ generators, but then $2 n-3 \geqslant\left(\begin{array}{l}n \\ 2\end{array}\right)$, which is possible only if $n \leqslant 3$.

Thus, at least for $n \geqslant 4, \operatorname{coker}(\psi)$ has nontrivial torsion concentrated in $m=$ maximal ideal. In particular, we obtain $\operatorname{Ext}_{R}^{i}(E, R)=0$ for $i=1, \ldots, n-3$. 
Consider the case $n=5: 0 \rightarrow L \rightarrow R^{7} \rightarrow E \rightarrow 0$. Here depth $L=2$, while depth $L^{*} \geqslant 3$, since $\operatorname{Ext}_{R}^{1}(L, R)=0$ implies $L^{*}$ is a third syzygy module. The $Z$-complex of $E$ is then

$$
0 \rightarrow R \otimes \tilde{S}[-3] \rightarrow L^{*} \otimes \tilde{S}[-2] \rightarrow L \otimes \tilde{S}[-1] \rightarrow \tilde{S} \rightarrow S(E) \rightarrow 0 .
$$

Applying the acyclicity lemma of [24] to the graded components (cf. [14]), it will follow that the complex is exact and $\operatorname{Sym}_{t}(E)$ is a torsion-free $R$-module, i.e. $S(E)$ is an integral domain. It has Krull dimension 9 and the grade of the maximal homogeneous ideal is $\geqslant 8$. We shall see, however (cf. $\S 6$ ), that $S(E)$ is not a Cohen-Macaulay ring.

EXAMPLE 4.5. Let $R$ be a Cohen-Macaulay integral domain and $E$ a torsion-free $R$-module of projective dimension two satisfying $\left(\mathscr{F}_{1}\right)$ and admitting a resolution $0 \rightarrow L \rightarrow R^{n} \rightarrow E \rightarrow 0$ with $\operatorname{rank}(L)=2$ (this is [30, (3.5)]).

The Z-complex of $E$ is then

$$
0 \rightarrow R \otimes \tilde{S}[-2] \rightarrow L \otimes \tilde{S}[-1] \rightarrow \tilde{S} \rightarrow S(E) \rightarrow 0 .
$$

$Z(E)$ is again acyclic and $S(E)$ is a Cohen-Macaulay integral domain.

ExAmple 4.6. Let $R$ be an integral domain and $E$ a torsion-free $R$-module. We consider another case in projective dimension two. In general, to obtain $S(E)=$ domain, one needs high depths in the modules $Z_{r}(E)$; in dimension two this may often be done since the projective dimensions of the exterior powers of $L=Z_{1}(E)$ are easier to estimate.

Consider the case of a module satisfying $\left(\mathscr{F}_{1}\right)$. In addition, to use the $Z$-complex it is convenient that $\wedge^{r} L=\left(\wedge^{r} L\right)^{* *}$ for each $r<\operatorname{rank}(L)$. One way to achieve it is to strengthen $\left(\widetilde{F}_{1}\right)$ in higher codimension by requiring

$$
v\left(E_{P}\right) \leqslant \frac{1}{2}(h t(P)+1)+\operatorname{rank}(E) \text { for each prime } P .
$$

We claim $Z(E)$ is acyclic and $S(E)$ is a domain.

We may assume we are dealing with a minimal resolution of $E$ and $E$ is not free. Since, from $\left(\mathscr{F}_{1}\right), v\left(E_{p}\right) \leqslant \mathrm{ht}(P)+\operatorname{rank}(E)-1$,

$$
v(E)-\operatorname{rank}(E)=\operatorname{rank}(L) \leqslant \operatorname{ht}(P)-1 \quad(P=\text { maximal ideal }) .
$$

To apply the acyclicity lemma as above and the underlying mapping cone property of the $Z$-complex (cf. [12, 30]), $\wedge^{r} L$ must have depth at least $r+1$ and be reflexive in the range $1<r<\operatorname{rank}(L)$. Since the projective dimension of $\wedge^{r} L$ is $r$, we must have $\operatorname{dim} R-r \geqslant r+1$, which is provided by $(*)$ above.

Problem. Determine necessary and sufficient conditions for a module of projective dimension two to admit an acyclic $Z$-complex.

Here is an example of a nice module of projective dimension two without an acyclic $Z$-complex. Let $R$ be a Cohen-Macaulay local ring and $\mathcal{K}(\mathbf{x} ; R)$ the Koszul complex associated to a system of parameters $\mathbf{x}=\left\{x_{1}, \ldots, x_{n}\right\}, n \geqslant 3$. Let $E=Z_{n-3}$, that is, consider the tail of $\mathscr{K}(\mathbf{x} ; R), 0 \rightarrow K_{n} \rightarrow K_{n-1} \rightarrow K_{n-2} \rightarrow E \rightarrow 0$. Alternatively, given the free module $R^{n}$, with basis $\left\{e_{1}, \ldots, e_{n}\right\}$, then $E$ is the cokernel of the mapping $R^{n} \rightarrow \wedge^{2} R^{n}$ defined by multiplication by $\zeta=\sum x_{i} e_{i}$.

Counting ranks, it follows that $E$ satisfies $\left(\mathscr{F}_{1}\right)$. As $Z_{n-2}(E)$ has depth 2 , it follows easily that $Z(E)$ is not acyclic if $n \geqslant 3$. 
EXAMPLE 4.7. In order to show the exactness of the $Z$-complex of a module, we have been striving for high depth for the coefficients $Z_{r}(E)$. Normally this entails that, locally, depth $Z_{r}(E) \geqslant r$. We shall shortly take another approach-that of acyclic sequences. We shall look, however, at a last example where estimation of the depth of $Z_{r}(E)$ is possible.

Let $R$ be a Gorenstein local ring and $I$ an ideal of grade $g \geqslant 1$, minimally generated by $n$ elements. The $Z_{r}(I)$ are then the modules of cycles of the associated Koszul complex. For $n-1 \geqslant r \geqslant n-g+1, Z_{r}=B_{r}=r$-boundaries. Since, in that range, pd $B_{r}=n-(r+1)$, depth $Z_{r}=d-n+r+1(d=\operatorname{dim} R)$.

When $I$ is a Cohen-Macaulay ideal, the depth of one extra $Z_{r}$ may be determined. Indeed, let us show that depth $Z_{n-g} \geqslant d-g+2$ (we may assume $g \geqslant 2$ ).

Consider the sequence

$$
0 \rightarrow B_{n-g} \rightarrow Z_{n-g} \rightarrow H_{n-g}(I ; R) \rightarrow 0 .
$$

Note that depth $B_{n-g}=d-g+1$, while $H_{n-g}(I ; R)$ is the canonical module of $R / I$ and thus has depth $d-g$. The exact sequence already says that depth $Z_{n-g} \geqslant d$ $-g$. To determine depth $Z_{n-g}$ we test the vanishing of the modules $\operatorname{Ext}_{R}^{i}\left(Z_{n-g}, R\right)$ for $i=g, g-1$ (cf. [21]). From the exact sequence we have the homology sequence

$$
\begin{aligned}
\operatorname{Ext}^{g-1}\left(H_{n-g}, R\right) & \rightarrow \operatorname{Ext}^{g-1}\left(Z_{n-g}, R\right) \rightarrow \operatorname{Ext}^{g-1}\left(B_{n-g}, R\right) \\
& \rightarrow \operatorname{Ext}^{g}\left(H_{n-g}, R\right) \rightarrow \operatorname{Ext}\left(Z_{n-g}, R\right) \rightarrow \operatorname{Ext}^{g}\left(B_{n-g}, R\right) .
\end{aligned}
$$

Here $\operatorname{Ext}^{g-1}\left(B_{n-g}, R\right)=R / I$, from the exactness of the corresponding tail of the Koszul complex. On the other hand, $\operatorname{Ext}^{g}\left(B_{n-g}, R\right)=\operatorname{Ext}^{g-1}\left(H_{n-g}, R\right)=0$, while $\operatorname{Ext}^{g}\left(H_{n-g}, R\right)=R / I$ since $R$ is a Gorenstein ring. Thus we have the exact sequence

$$
0 \rightarrow \operatorname{Ext}^{g-1}\left(Z_{n-g}, R\right) \rightarrow R / I \stackrel{\phi}{\rightarrow} R / I \rightarrow \operatorname{Ext}^{g}\left(Z_{n-g}, R\right) \rightarrow 0 .
$$

Localizing at primes of height $g$ and $g+1$, we get that $\phi$ is an isomorphism since $Z_{n-g}$ is a second syzygy module. Thus $\phi$ is an isomorphism and the desired assertion follows.

The first instance of interest to which this applies is when $n=g+2$. One obtains that depth $Z_{2}=d-g+2$. Since depth $Z_{1}=d-g+2$, we conclude that the $H_{i}(I ; R)$ are Cohen-Macaulay modules. (See [2] for the original proof.)

For $n=g+3$, we get depth $Z_{3}=d-g+2$, depth $Z_{1}=d-g+2$ and depth $Z_{2} \geqslant 2$. If the ideal $I$ satisfies $\left(\mathscr{F}_{0}\right)$, it will follow that the complex $Z(I)$ is acyclic [12]. Thus, for the ideal generated by the $2 \times 2$ minors of a generic matrix,

$$
\left[\begin{array}{llll}
x_{1} & x_{2} & x_{3} & x_{4} \\
y_{1} & y_{2} & y_{3} & y_{4}
\end{array}\right]
$$

$Z(I)$ is acyclic.

In case $n=g+3$ if depth $Z_{2} \geqslant 3$ and $I$ satisfies ( $\left.\widetilde{F}_{1}\right)$, we have that $S(I)$ and the Rees algebra $R(I)=R[I T]$ coincide [12]. In the above example, because of the Plücker relations, we must have depth $Z_{2}=2$. 
Now suppose $I$ is the ideal generated by the $2 \times 2$ minors of the generic symmetric matrix

$$
\left[\begin{array}{lll}
x_{11} & x_{12} & x_{13} \\
x_{12} & x_{22} & x_{23} \\
x_{13} & x_{23} & x_{33}
\end{array}\right] .
$$

Here $n=g+3$ and $I$ satisfies $\left(\mathscr{F}_{1}\right)$. We do not know whether depth $Z_{3} \geqslant 3$.

5. Acyclicity. We begin by recalling the notions that play for the Z-complex the role of acyclic sequences.

Definition 5.1. Suppose $\mathbf{x}=\left\{x_{1}, \ldots, x_{n}\right\}$ is a sequence of elements in a ring $R$. The sequence $\mathbf{x}$ is called a:

(a) $d$-sequence if:

$\left(\mathrm{a}_{1}\right) \mathbf{x}$ is a minimal generating set of the ideal $I=(\mathbf{x})=\left(x_{1}, \ldots, x_{n}\right)$;

$\left(\mathrm{a}_{2}\right)\left(x_{1}, \ldots, x_{i}\right): x_{i+1} x_{k}=\left(x_{1}, \ldots, x_{i}\right): x_{k}$, for $i=0, \ldots, n-1$ and $k \geqslant i+1$;

(b) proper sequence if $x_{i+1} H_{j}\left(x_{1}, \ldots, x_{i} ; R\right)=0$ for $i=0, \ldots, n-1, j>0$, where $H_{j}\left(x_{1}, \ldots, x_{i} ; R\right)$ denotes the Koszul homology associated to the initial subsequence $\left\{x_{1}, \ldots, x_{i}\right\}$.

REMARKS. (i) The relationship between (a) and (b) is, broadly, the following: Each $d$-sequence is a proper sequence and the linear forms in $S(I)$ corresponding to a proper sequence $\mathbf{x}$ generate a $d$-sequence relative to the ring $S(I)$ (cf. [14, (12.10)]).

(ii) In (b) it suffices to consider $j=1$ [19].

(iii) In $(\mathrm{a}),\left(\mathrm{a}_{2}\right)$ already embodies a measure of minimality. For instance, assume $I=\left(x_{1}, \ldots, x_{n}\right)$ is an ideal of grade $k$; then $x_{1}, \ldots, x_{k}$ is, as seen directly from $\left(\mathrm{a}_{2}\right)$, a regular sequence. Partly for this reason, we shall at times blur the definition by considering $\left(\mathrm{a}_{2}\right)$ alone.

For quick reference we quote the following criterion [14, (12.9), 19].

Proposition 5.2. Let $R$ be a Noetherian local ring with infinite residue field and $E$ a finitely generated $R$-module. The following are equivalent:

(a) $Z(E)$ is acyclic.

(b) $S_{+}$is generated by a d-sequence of linear forms of $S(E)$.

Let us indicate how these conditions can be realized. For simplicity assume $R$ is a domain. A Bourbaki sequence (Bs) is an exact sequence of an $R$-module $0 \rightarrow F \rightarrow E$ $\rightarrow I \rightarrow 0$, where $F$ is a free module and $I$ is an ideal.

Assume that in such a sequence $I$ is generated by a $d$-sequence. The symmetric algebra $S(I)$ in this case coincides with the Rees algebra, and therefore will be an integral domain [17, 32]. Since $S(I)=S(E) /(F)((F)=$ ideal of $S(E)$ generated by the forms in $F),(F)$ will be a prime ideal of height $=\operatorname{rank}(F)$. It will follow from [5] that any basis of $F$ will generate a regular sequence in $S(E)$. Putting together a generating set of $E$ from a basis of $F$ and elements mapping to the $d$-sequence in $I$, we conclude that the irrelevant ideal of $S(E)$ is indeed generated by a $d$-sequence, whence $Z(E)$ will be acyclic. (The local and infinite residue field hypotheses are needed in the other direction only.)

In this construction, if $S(I)$ is integrally closed and $R$ is a Japanese ring, then $S(E)$ will also be integrally closed by Hironaka's lemma. 
One of our purposes here is to prove a broad converse that expresses certain modules $E$ with $Z(E)$ acyclic in terms of Bourbaki sequences.

Assume from this point on that $R$ is a local ring with infinite residue field and let $E$ be a finitely generated $R$-module. The following lemmas provide the means to check whether a sequence generating $S_{+}$is a $d$-sequence.

LemMa 5.3. Let $x_{1}, \ldots, x_{n}$ be 1-forms generating $S_{+}$. The following conditions are equivalent:

(a) $\left\{x_{1}, \ldots, x_{n}\right\}$ is a d-sequence.

(b) $H_{1}\left(x_{1}, \ldots, x_{i} ; S(E)\right)_{2}=0$ for $i=1, \ldots, n$.

(c) $H_{1}\left(x_{1}, \ldots, x_{i} ; S(E)\right)_{k}=0$ for $i=1, \ldots, n$, and $k \geqslant 2$.

Proof. (a) $\Leftrightarrow$ (c) is $[14,(12.7)]$.

(b) $\Rightarrow$ (c) We proceed by induction on $k$. Let $k>2$ and suppose

$$
H_{1}\left(x_{1}, \ldots, x_{i} ; S(E)\right)_{k-1}=0 \text { for } i=1, \ldots, n .
$$

From the long exact sequence of Koszul complexes (cf. [22]),

$$
H_{1}\left(x_{1}, \ldots, x_{i} ; S(E)\right)_{k-1} \stackrel{x_{i+1}}{\rightarrow} H_{1}\left(x_{1}, \ldots, x_{i} ; S(E)\right)_{k} \rightarrow H_{1}\left(x_{1}, \ldots, x_{i+1} ; S(E)\right)_{k},
$$

and we inductively conclude that for each $i$,

$$
H_{1}\left(x_{1}, \ldots, x_{i} ; S(E)\right)_{k} \rightarrow H_{1}\left(x_{1}, \ldots, x_{n} ; S(E)\right)_{k}
$$

is injective. But this last module vanishes for $k>1$ [19].

Let $E$ be a module with $\operatorname{rank}(E)=e$. In general, for the symmetric algebra $S(E)$, one has grade $S_{+} \leqslant \operatorname{rank}(E)$.

LEMmA 5.4. If $Z(E)$ is acyclic, then grade $S_{+}=\operatorname{rank}(E)$. In particular, if $\left\{x_{1}, \ldots\right.$, $\left.x_{n}\right\}$ is a d-sequence of 1-forms generating $S_{+}$then $\left\{x_{1}, \ldots, x_{e}\right\}$ is a regular sequence on $S(E)$.

Proof. Map a polynomial ring $\tilde{S}=R\left[e_{1}, \ldots, e_{n}\right]$ onto a generating set $y_{1}, \ldots, y_{n}$ of 1 -forms of $S(E)$. The $Z$-complex of $E$ has length $n-e$ and, for each component, $\operatorname{Tor}_{i} \tilde{S}\left(\tilde{S} /(\mathbf{e}), Z_{r}(E) \otimes S[-r]\right)=0$ and $i>0$. If $Z(E)$ is exact it can be used to read the grade of

$S_{+}=(\mathbf{e})-\operatorname{depth} S(E)=n-\sup \left\{i \mid \operatorname{Tor}_{i} \tilde{S}(\tilde{S} /(\mathbf{e}), S(E)) \neq 0\right\}=n-(n-e)=e$.

The last assertion follows from an earlier remark.

LemmA 5.5. Let $\left\{x_{1}, \ldots, x_{n}\right\}$ be a sequence of 1-forms in $S_{+}$. Suppose the first $r$ elements form a regular sequence. The following conditions are equivalent:

(a) $\left\{x_{1}, \ldots, x_{n}\right\}$ is a d-sequence.

(b) $\left\{x_{r+1}^{*}, \ldots, x_{n}^{*}\right\}$ is a d-sequence on $S^{*}=S(E) /\left(x_{1}, \ldots, x_{r}\right)$.

Proof. The assertion follows from (5.3) and the isomorphism of graded modules $H_{1}\left(x_{r+1}^{*}, \ldots, x_{i}^{*} ; S^{*}\right)=H_{1}\left(x_{1}, \ldots, x_{i} ; S(E)\right)$, valid for all $i>r$.

We can now prove the relationship between the acyclicity of $Z(E)$ and the special representations of $E$ in terms of Bourbaki sequences. 
THEOREM 5.6. Let $R$ be a local ring with infinite residue field. Suppose $E$ is a finitely generated torsion-free $R$-module and either (i) $\operatorname{pd} E<\infty$, or (ii) $R$ is a normal domain. Then the following conditions are equivalent:

(a) $Z(E)$ is acyclic.

(b) $E$ admits a Bourbaki sequence $0 \rightarrow F \rightarrow E \rightarrow I \rightarrow 0$ such that:

$\left(b_{1}\right) F$ is generated by elements which form a regular sequence of 1-forms on $S(E)$.

$\left(b_{2}\right) I$ is generated by a proper sequence.

Proof. (b) $\Rightarrow$ (a) Note that a module $E$ as above has a well-defined rank, say $\operatorname{rank}(E)=e$. Pick generators $\left\{x_{1}, \ldots, x_{e-1}\right\}$ of $F$ which form a regular sequence on $S(E)$ and pick elements $\left\{x_{e}, \ldots, x_{n}\right\}$ in $E$ whose images in $I$ form a proper sequence; $\left\{x_{1}, \ldots, x_{n}\right\}$ is a system of generators of $S_{+}$. By $[14,(12.10)]$, the elements $\left\{x_{3}, \ldots, x_{n}\right\}$ form a $d$-sequence on $S(I)$; hence, by $5.5,\left\{x_{1}, \ldots, x_{n}\right\}$ is a $d$-sequence on $S(E)$. The assertion now follows from 5.2.

(a) $\Rightarrow$ (b) We proceed by induction on $e$. If $e=1$, then $E$ is isomorphic to an ideal and 5.2 applies.

Suppose then $\operatorname{rank}(E)=e>1$. Since $Z(E)$ is acyclic we have by 5.4 that grade $S_{+}=e>1$. Hence we can find $x \in E$, which is a nonzero divisor on $S(E)$. Our aim is to find an element $x$ with the additional property that $E / R x$ is still torsion-free. We then apply the induction hypothesis to $E / R x$ and the theorem will be proved.

Denote by $x^{*}$ the image of an element $x$ in $E / m E(m=$ maximal ideal of $R$ ). To find $x$ which is regular on $S(E)$ amounts to finding $x$ such that $x^{*} \notin X$, where $X \subset E / \mathrm{m} E$ is a finite union of proper linear subspaces which are determined by Ass $(S(E))$. The proof will be completed by the next lemma.

Lemma 5.7. Suppose $E$ satisfies the conditions of the theorem and $\operatorname{rank}(E)=e>1$. Let $X$ be a finite union of proper linear subspaces of $E / \mathrm{m} E$. There exists $x \in E$ such that

(i) $E / R x$ is torsion-free,

(ii) $x^{*} \notin X$.

Proof. (i) is satisifed if $x$ is $P$-basic for all primes $P$ of $R$ with depth $R_{P} \leqslant 1$. (ii) will be satisfied if $\lambda_{i}(x)$ is m-basic for $i=1, \ldots, k$, where $\lambda_{i}: E \rightarrow L_{i}$ are epimorphisms and $X=-L_{i}$. Such an element exists according to [4, (2.4)].

We now relate the acyclicity of $Z(E)$ with the resolution of $S(E)$ as an $\tilde{S}$-module.

Let $\{\mathcal{G}, \partial\}$ be a minimal $\tilde{S}$-resolution of $S(E)$, i.e. $\mathcal{G}$ is a (graded) $\tilde{S}$-projective resolution of $S(E)$ and $\partial(\mathcal{G}) \subset\left(\mathrm{m} \tilde{S}+\tilde{S}_{+}\right) \mathcal{G}$. We define the filtration $\mathscr{F}_{-i} \mathcal{G}$ on $\mathcal{G}$ by

$$
\left(\mathscr{F}_{-i} \mathcal{G}\right)_{j}=\bigoplus_{a_{j k} \leqslant i} \tilde{S}\left[-a_{j k}\right] .
$$

It is clear that for each $i, \mathscr{F}_{-i+1} \mathcal{G}$ is a subcomplex of $\mathscr{F}_{-i} \mathcal{G}$ and $\mathscr{F}_{-i} \mathcal{G} / \mathscr{F}_{-i+1} \mathcal{G}=\mathscr{L}_{i} \otimes$ $\tilde{S}[-i]$, where $\mathcal{L}_{i}$ is a complex of $R$-modules.

THEOREM 5.8. The following conditions are equivalent:

(a) $Z(E)$ is acyclic.

(b) All the complexes $\mathfrak{L}_{i}$ are acyclic. 
If the equivalent conditions hold, then $\mathcal{L}_{i}$ is a minimal $R$-free resolution of $H_{i}\left(S_{+} ; S(E)\right)_{i}=Z_{i}(E)$ shifted $i$ steps to the left. In particular, one has the relation of Betti numbers

$$
\beta_{i}^{\tilde{S}}(S(E))=\sum_{j} \beta_{i-j}^{R}\left(Z_{j}(E)\right) .
$$

Proof. For any graded $\tilde{S}$-module $M$, put $M^{*}=M / \tilde{S}_{+} M$. We have the isomorphism of graded modules

$$
H_{i}\left(S_{+} ; S(E)\right)=\operatorname{Tor}_{i}^{\tilde{S}}(R, S(E))=H_{i}\left(\mathcal{G}^{*}\right),
$$

and

$$
\mathcal{G}^{*}=\bigoplus_{i \geqslant 0}\left(\mathscr{F}_{-i} \mathcal{G} / \mathscr{F}_{-i+1} \mathcal{G}\right)^{*}=\bigoplus_{i \geqslant 0} \mathcal{L}_{i}
$$

where the $\mathcal{L}_{i}$ are complexes of $R$-modules $L_{i k}$ which, considered as $\tilde{S}$-modules, are concentrated in degree $i$. It follows that

$$
H_{i}\left(S_{+} ; S(E)\right)_{j}=H_{i}\left(\varrho_{j}\right) \text {. }
$$

The equivalence of (a) and (b) now follows from 3.2. The additional assertions of the theorem follow trivially.

Remark. If $\beta_{i j}$ denotes the $(R-) j$ th Betti number of $H_{i}\left(S_{+} ; S(E)\right)_{i}$, and $Z(E)$ is acyclic, then the $\tilde{S}$-resolution of $S(E)$ looks like

$$
\begin{aligned}
\cdots \bigoplus_{j=1}^{i} \tilde{S}[-j]^{\beta_{j, i-j}} & \rightarrow \cdots \rightarrow \tilde{S}[-2]^{\beta_{2,0}} \oplus \tilde{S}[-1]^{\beta_{1,1}} \\
& \rightarrow \tilde{S}[-1]^{\beta_{1,0}} \rightarrow \tilde{S} \rightarrow S(E) \rightarrow 0 .
\end{aligned}
$$

6. Cohen-Macaulay and Gorenstein symmetric algebras. Throughout this section we assume $R$ is a Cohen-Macaulay ring. We focus on obtaining symmetric algebras which are Cohen-Macaulay in the context of acyclic $Z$-complexes. The main point that emerges is strict depth conditions on the coefficient modules $Z_{i}(E)$ of $Z(E)$.

Since the $Z_{i}(E)$ are Koszul homology modules, several of the methods used to prove acyclicity of $Z(E)$ require high depth on such modules. In [12], for instance, for an ideal $I$ we required that $H_{i}(I ; R)$ be Cohen-Macaulay for all $i$ although the proofs themselves made different demands on the various $H_{i}(I ; R)$. We now introduce a sliding condition that is closer to the needs of the $Z$-complex.

Let $I$ be an ideal of the Cohen-Macaulay local ring; say $\mathbf{a}=\left\{a_{1}, \ldots, a_{n}\right\}$ is a generating set for $I$. We shall look at conditions of the type depth $H_{i}(\mathbf{a} ; R) \geqslant d-n+i+k$ for all $i$.

Here $d=\operatorname{dim} R, k=$ fixed integer and, as usual, $\operatorname{depth}(0)=\infty$. In case $I$ is a homogeneous ideal of a graded $R$-algebra $A$, we will restrict to the $i$ th component of $H_{i}(I ; A)$ the discussion below.

Let us remark on some elementary properties of this notion-generally denoted sliding depth. 
(i) First we claim $\left(\varsigma \mathscr{D}_{k}\right)$ is independent of the generating set a. To see this it is enough to compare $\left(\delta \mathscr{D}_{k}\right)$ for two generating sets a and $\mathbf{a}^{\prime}=\{\mathbf{a}, 0\}$. Suppose $\left(\delta \mathscr{D}_{k}\right)$ holds for a: Then, for each $i$, we have

$$
H_{i}\left(\mathbf{a}^{\prime} ; R\right)=H_{i}(\mathbf{a} ; R) \oplus H_{i-1}(\mathbf{a} ; R),
$$

therefore depth $H_{i}\left(\mathbf{a}^{\prime} ; R\right) \geqslant d-n+(i-1)+k$, as required. Conversely, if $\left(\delta \mathcal{O}_{k}\right)$ holds for $\mathbf{a}^{\prime}$ but not for $\mathbf{a}$, let $i$ be highest so that the condition fails, that is, pick $i$ largest with depth $H_{i}(\mathbf{a} ; R)<d-n+i-1+k$. But writing $(*)$ for $i+1$ instead of $i$, we would then get a contradiction.

(ii) If $R$ is a Cohen-Macaulay ring, the localizations of $I$ will have $\left(\delta \mathcal{D}_{k}\right)$ if it holds true for the maximal ideals. More generally, let $R$ be a Cohen-Macaulay local ring of dimension $d$ and let $G$ be a finitely generated $R$-module. Assume depth $G \geqslant d$ $-r$. Then for any prime ideal $P$, depth $G_{P} \geqslant \operatorname{ht}(P)-r$. This is clear if $R$ is regular, since $d-\operatorname{depth} G$ is then the projective dimension of $G$ which does not increase under localization. But the underlying idea also works in the more general case: Assume first that $R$ admits a canonical module $\omega$. In this case

$$
d-\operatorname{depth} G=\sup \left\{j \mid \operatorname{Ext}_{R}^{j}(G, \omega) \neq 0\right\} .
$$

Since the localization $\omega_{P}$ is a canonical module for $R_{P}$, the assertion again follows.

For general Cohen-Macaulay rings a simple argument with its m-adic completion yields the same conclusion.

In the case of an $R$-module $E$, we define $\left(S \mathscr{D}_{k}\right)$ on $S(E)$ as

$$
\operatorname{depth} H_{i}\left(S_{+} ; S(E)\right)_{i} \geqslant d-n+i+k, \quad i \geqslant 0 \text {. }
$$

The unqualified sliding depth condition will refer to the case $k=\operatorname{rank}(E)$.

(iii) Despite the appearance, there is no ambiguity regarding the sliding depth on the ideal $I$ : whether one means $H_{i}(I ; R)$ or $H_{i}\left(S_{+} ; S(I)\right)_{i}$. Indeed, as noted the latter is the corresponding module of cycles in the Koszul complex $\mathscr{K}(\mathbf{a} ; R)$ associated to a. Thus, with $B_{i}$ and $Z_{i}$ denoting the boundaries and cycles of $\mathscr{K}(\mathbf{a} ; R)$, to show the equivalence of the two conditions we simply chase depths in the sequences (cf. [12]) $0 \rightarrow Z_{i+1} \rightarrow K_{i+1} \rightarrow B_{i} \rightarrow 0$ and $0 \rightarrow B_{i} \rightarrow Z_{i} \rightarrow H_{i}(\mathbf{a} ; R) \rightarrow 0$.

EXAMPLE 6.1. It is easy to see that if $I$ is generated by a proper sequence $\mathbf{x}=\left\{x_{1}, \ldots, x_{n}\right\}$ and satisfies sliding depth, then for any initial subsequence $\mathbf{x}^{\prime}=$ $\left\{x_{1}, \ldots, x_{m}\right\}, m<n, J=\left(\mathbf{x}^{\prime}\right)$ will satisfy the same sliding depth condition. This is obtained directly from the Koszul homology sequences associated to $\mathbf{x}^{\prime}$ and $\left\{\mathbf{x}^{\prime}, x_{m+1}\right\}$ and the properness. As a consequence, for any of the ideals known to have to Cohen-Macaulay Koszul homology (or, strongly Cohen-Macaulay in the terminology of Huneke) and is generated by proper sequences, one obtains various other ideals with sliding depth and not always Cohen-Macaulay.

Another way to obtain ideals of height one satisfying sliding depth is the following. Let $I$ satisfy sliding depth and assume it is generated by a $d$-sequence. By Remark (iii) above it follows that the ideal $S_{+}$of the Rees algebra $S(I)$ will satisfy sliding depth as well. 
The naturality of $\left(\delta \mathscr{D}_{e}\right)$ is brought into relief by

THEOREM 6.2. Let $R$ be a Cohen-Macaulay local ring and $E$ a finitely generated $R$-module of $\operatorname{rank}(E)=e$. The following conditions are equivalent:

(a) $Z(E)$ is acyclic and $S(E)$ is Cohen-Macaulay.

(b) E satisfies sliding depth $\left(\left(\mathcal{S} \mathscr{Q}_{e}\right)\right)$ and $\left(\mathscr{F}_{0}\right)$.

Note that $\left(\mathscr{F}_{0}\right)$ and $\left(\delta \mathscr{D}_{e}\right)$ together place rather strict bounds on the coefficient modules $Z_{r}(E)$ of the complex $Z(E)$. Indeed, $\left(\mathscr{F}_{0}\right)$ implies $d-n+e \geqslant 0, d=$ $\operatorname{dim} R, n=v(E)$, while $\left(\delta \mathscr{Q}_{e}\right)$ requires depth $Z_{r}(E) \geqslant(d-n+e)+r$ for all $r$. We also recall that $\left(\mathscr{F}_{0}\right)$ just means $S(E)$ has its expected Krull dimension, that is, $\operatorname{dim} S(E)=\operatorname{dim} R+\operatorname{rank}(E)$.

Proof. (b) $\Rightarrow$ (a) The exactness of the complex $Z(E)$ follows as in [14]. A simple depth-counting argument shows depth $S(E) \geqslant d+\operatorname{rank}(E)$, so $S(E)$ is CohenMacaulay.

(a) $\Rightarrow$ (b) Since $S(E)$ is Cohen-Macaulay, we have $\operatorname{dim} S(E)=\operatorname{dim} R+\operatorname{ht}\left(S_{+}\right)$ $=\operatorname{dim} R+\operatorname{rank}(E)$, as in 5.4.

We may assume $R$ admits a canonical module $\omega_{R}$. Note that $\omega_{\tilde{S}}=\omega_{R} \otimes \tilde{S}$. If $M$ is an $\tilde{S}$-module, we put $M^{\nu}=\operatorname{Hom}_{\tilde{S}}\left(M, \omega_{\tilde{S}}\right)$. We also abbreviate the notations of 5.8 and write $\mathcal{F}_{-i}$ instead of $\mathscr{F}_{-i} \mathcal{G}$.

Claim. $H^{j}\left(\left(\mathscr{F}_{-i} / \mathscr{F}_{-i+1}\right)\right)=0$ for $j>l=n-e$.

Let us first see what this claim entails. If $\mathcal{L}_{i}$ is a minimal $R$-free resolution of $H_{i}\left(S_{+} ; S(E)\right)_{i}=Z_{i}(E)$, then from 5.8,

$$
\mathscr{F}_{-i} / \mathscr{F}_{-i+1}=\mathcal{L}_{i}[-i] \otimes \tilde{S}[-i] .
$$

Therefore

$$
\text { (\#) } \begin{aligned}
& H^{j}\left(\operatorname{Hom}_{\tilde{S}}\left(\varrho_{i}[-i]\right) \otimes_{R} \tilde{S}[-i], \omega_{\tilde{S}}\right)=H^{j}\left(\operatorname{Hom}_{R}\left(\varrho_{i}[-i], \omega_{R}\right)\right) \otimes_{R} \tilde{S}[i] \\
&=H^{j-i}\left(\operatorname{Hom}_{R}\left(\varrho_{i}, \omega_{R}\right)\right) \otimes_{R} \tilde{S}[i]=\operatorname{Ext}_{R}^{j-i}\left(Z_{i}(E), \omega_{R}\right) \otimes \tilde{S}[i] .
\end{aligned}
$$

If the last module vanishes, then depth $Z_{i}(E) \geqslant d-(j-i)+1$; in particular, this holds for $j>n-e$, from which sliding depth follows.

PROOF OF THE CLAIM. We show by induction on $r$ that

$$
\text { (\#) } \quad H^{j}\left(\left(\mathcal{F}_{-l+r} / \mathscr{F}_{-l+r+1}\right)^{\nu}\right)=H^{j}\left(\left(\mathscr{F}_{-l+r}\right)^{\nu}\right)=0 \text { for } j>l \text {. }
$$

$r=0:$ Since $\mathscr{F}_{-l}=\mathcal{G}$ and $S$ is Cohen-Macaulay, we have

$$
H^{j}\left(\left(\mathscr{F}_{-l}\right)^{\nu}\right)=\operatorname{Ext}_{\tilde{S}}^{j}\left(S, \omega_{\tilde{S}}\right)=0 \text { for } j>l \text {. }
$$

The exact sequence $0 \rightarrow \mathscr{F}_{-l+1} \rightarrow \mathscr{F}_{-l} \rightarrow \mathscr{F}_{-l} / \mathscr{F}_{-l+1} \rightarrow 0$ gives rise to the homology exact sequence

$$
H^{j-1}\left(\left(\mathscr{F}_{-l+1}\right)^{\sim}\right) \stackrel{\phi}{\rightarrow} H^{j}\left(\left(\mathscr{F}_{-l} / \mathscr{F}_{-l+1}\right)^{\nu}\right) \rightarrow H^{j}\left(\left(\mathscr{F}_{-l}\right)^{\llcorner}\right)
$$

Suppose $j>l$; then $\phi$ is surjective. Now $H^{j}\left(\left(\mathscr{F}_{-l} / \mathscr{F}_{-l+1}\right) \zeta\right)$ is generated by elements of degree $-l$, see $(\#)$, while $H^{j-1}\left(\left(\mathscr{F}_{-l+1}\right)\right)$ is generated by elements of degree $\geqslant-l$ +1 . It follows that $H^{j}\left(\left(\mathscr{F}_{-l} / \mathscr{F}_{-l+1}\right)\right)=0$.

The proof of the induction step is similar. 
COROLlary 6.3. Let $R$ be a Cohen-Macaulay integral domain and $E$ a finitely generated $R$-module satisfying $\left(S_{\Omega_{e}}\right)$ and $\left(\mathscr{F}_{1}\right)$. Then $S(E)$ is a Cohen-Macaulay integral domain.

COROllary 6.4. Let $R$ be a Cohen-Macaulay ring and I an ideal generated by a $d$-sequence. The following conditions are equivalent:

(a) The Rees algebra $R(I)=\oplus_{t \geqslant 0} I^{t}$ is Cohen-Macaulay.

(b) The associated graded algebra $\operatorname{gr}_{I}(R)=\bigoplus_{t \geqslant 0} I^{t} / I^{t+1}$ is Cohen-Macaulay.

(c) I satisfies sliding depth.

With the notations of 6.2 , we have

TheOREm 6.5. Suppose $Z(E)$ is acyclic and $S=S(E)$ is Cohen-Macaulay. Then:

(a) $\omega_{S} / S_{+} \omega_{S}=\oplus_{i=0}^{l} \operatorname{Ext}_{R}^{l-i}\left(Z_{i}(E), \omega_{R}\right)$.

(b) The following conditions are equivalent:

$\left(\mathrm{b}_{1}\right) S$ is Gorenstein.

$$
\operatorname{depth} Z_{i}(E) \geqslant d-n+e+i+1 \text { for } i=0, \ldots, l-1,
$$

and $\operatorname{Hom}_{R}\left(Z_{l}(E), \omega_{R}\right)=R$.

Proof. (b) follows directly from (a). To prove (a), let $\omega_{S}=\operatorname{Ext}_{\tilde{S}}^{\prime}\left(S, \omega_{\tilde{S}}\right)$ be the canonical module of $S$. We use the results of (6.2): By (\# \#) we have exact sequences

$$
H^{l-1}\left(\left(\mathscr{F}_{-i+1}\right)^{\swarrow}\right) \stackrel{\phi_{i}}{\rightarrow} H^{l}\left(\left(\mathscr{F}_{-i} / \mathscr{F}_{-i+1}\right)^{\nu}\right) \stackrel{\psi_{i}}{\rightarrow} H^{l}\left(\left(\mathscr{F}_{-i}\right)^{\swarrow}\right) \rightarrow H^{l}\left(\left(\mathscr{F}_{-i+1}\right)^{\swarrow}\right) \rightarrow 0 .
$$

Denote again by ' $*$ ' the reduction $S \rightarrow S / S_{+}$. We obtain the exact sequence of $R$-modules

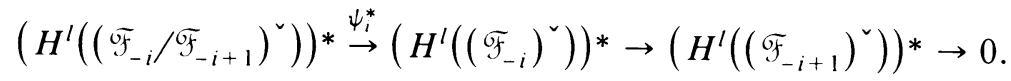

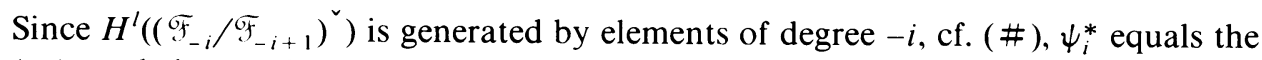
$(-i)$-graded part of $\psi_{i}$ :

$$
\left(\psi_{i}\right)_{-i}: H^{l}\left(\left(\mathscr{F}_{-i} / \mathscr{F}_{-i+1}\right)^{\nu}\right)_{-i} \rightarrow H^{l}\left(\left(\mathscr{F}_{-i}\right)^{\nu}\right)_{-i} .
$$

Since, on the other hand,

$$
\operatorname{Image}\left(\phi_{i}\right) \subset \bigoplus_{j \geqslant-i+1} H^{l}\left(\left(\mathscr{F}_{-i} / \mathscr{F}_{-i+1}\right)^{2}\right)_{j},
$$

it follows that $\left(\psi_{i}\right)_{-i}=\psi_{i}^{*}$ is injective. Hence we obtain the exact sequence

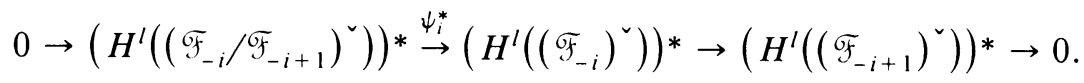

Arguing with degrees, we see that this exact sequence splits, which gives

$$
\left(\omega_{s}\right)^{*}=\left(\operatorname{Ext}_{\tilde{S}}^{l}\left(S, \omega_{\tilde{S}}\right)\right)^{*}=\left(H^{l}\left(\left(\mathscr{F}_{-l}\right)^{\nu}\right)\right)^{*}=\bigoplus_{i}\left(H^{l}\left(\left(\mathscr{F}_{-i} / \mathscr{F}_{-i+1}\right)^{\nu}\right)\right)^{*} \text {. }
$$

The assertion now follows from (\#) in the proof of 6.2 .

Corollary 6.6. Let $R$ be a Cohen-Macaulay ring. If I is a strongly Cohen-Macaulay ideal and $v\left(I_{P}\right) \leqslant \mathrm{ht}(P)+1$ for all primes $P$, then $S=S(I)$ is Cohen-Macaulay. If $R$ admits a canonical module and $\mathrm{ht}(I)=g \geqslant 2$, then $\omega_{S} / S_{+} \omega_{S}=\omega_{R} \oplus\left(\omega_{R} / I \omega_{R}\right)^{g-2}$. 
Proof. See [13] for the acyclicity of $Z(E)$. If $I$ is strongly Cohen-Macaulay and $Z$ denotes the cycles of a Koszul complex associated with $I$, then $(n=v(I), d=\operatorname{dim} R)$

$$
\operatorname{depth} Z_{i}(E) \begin{cases}\geqslant d-g+2 & \text { for } i=1, \ldots, n-g+1, \\ =d+i-(n-1) & \text { for } i=n-g+1, \ldots, n-1 .\end{cases}
$$

It follows that

$$
\operatorname{Ext}_{R}^{l-i}\left(Z_{i}(E), \omega_{R}\right)=\operatorname{Ext}_{R}^{n-1-i}\left(Z_{i}(E), \omega_{R}\right)=0 \quad(l=n-1)
$$

for $i=1, \ldots, n-g$, since $(n-1-i)+(d-g+2) \geqslant d+1$ for $i$ in the first range. For $i \geqslant n-g+1$, we have the exact sequences

$$
0 \rightarrow K_{n} \rightarrow K_{n-1} \rightarrow \cdots \rightarrow K_{i+1} \rightarrow Z_{i}(E) \rightarrow 0
$$

and therefore,

$$
\operatorname{Ext}_{R}^{n-1-i}\left(Z_{i}(E), \omega_{R}\right)=\omega_{R} / I \omega_{R} .
$$

The assertion now follows from $6.5(\mathrm{a})$.

COROLlary 6.7 [27]. Let $R$ be a Cohen-Macaulay ring and I an ideal containing regular elements. If $S(I)$ is Gorenstein then $R$ is Gorenstein and $\operatorname{ht}(I) \leqslant 2$.

Proof. By 6.4, $S(I)$ Gorenstein implies $\operatorname{Hom}_{R}\left(Z_{l}(I), \omega_{R}\right)=R$; since $Z_{l}(I)=R$, $R$ is Gorenstein. After localizing at a minimal prime of $I$, we may assume $I$ is primary relative to the maximal ideal. Since $S(I)$ is Cohen-Macaulay, we have $v(I) \leqslant \operatorname{dim} R+1[13]$. Thus we may apply 6.6 since $I$ is now strongly CohenMacaulay.

COROLlary 6.8. Let $R$ be a regular local ring and $E$ a finitely generated $R$-module. The following conditions are equivalent:

(a) $Z(E)$ is acyclic and $S(E)$ is Gorenstein.

(b) $E$ has projective dimension at most one and satisfies $\left(\mathscr{F}_{0}\right)$.

Proof. (a) $\Rightarrow$ (b) $\left(\mathscr{F}_{0}\right)$ follows from the Cohen-Macaulayness of $S(E)$ [13]. As $Z(E)$ is acyclic, we have (cf. §5)

$$
\mathcal{G}_{i}=\bigoplus_{j=1}^{i} \tilde{S}[-j]^{\beta_{j, i-j}}
$$

Since $S(E)$ is Gorenstein, $\mathcal{G}_{l}=\tilde{S}[-l]$ and $\mathcal{G}$ is self-dual. It follows that $\mathcal{G}_{i}=\tilde{S}[-i]^{\beta_{i, 0}}$ and, hence, all the modules $Z_{i}(E)$ are free. In particular, $Z_{1}(E)$ is free and pd $E \leqslant 1$.

(b) $\Rightarrow$ (a) follows from 4.1.

7. Reflexive symmetric algebras. We are now concerned with the symmetric algebra of a module $E, S(E)=\oplus \operatorname{Sym}_{t}(E)$, with each $\operatorname{Sym}_{t}(E)$ a reflexive module. When $R$ is a Cohen-Macaulay ring we have already identified a necessary condition, namely $\left(\mathscr{F}_{2}\right)$. If $E$ is a module of projective dimension one, $0 \rightarrow R^{m} \rightarrow R^{n} \rightarrow E \rightarrow 0$, $\left(\mathscr{F}_{2}\right)$ is also a sufficient condition [30]. The paucity of other classes of examples has led us to state

Conjecture 7.1. Let $R$ be a regular local and let $E$ be a finitely generated $R$-module. If $S(E)$ is reflexive, then pd $E \leqslant 1$. 
Stated otherwise it says that if $S(E)$ is factorial then $S(E)$ must be a complete intersection. In this section we shall describe some of the emerging evidence in support of this conjecture. We shall often depart from the hypothesis $R=$ regular, but the finiteness of the projective dimension will be kept.

Proposition 7.2. Let $R$ be a Cohen-Macaulay ring and let $E$ be a finitely generated $R$-module. If $E$ satisfies $\left(\widetilde{\mathscr{F}}_{2}\right)$, then $\mathrm{pd} E \neq 2$.

Proof. Assume otherwise; pick $R$ local with lowest possible dimension, that is, we may assume $\operatorname{pd}_{R_{P}} E_{P} \leqslant 1$ for each prime $P \neq \mathrm{m}=$ maximal ideal of $R$. Let

$$
0 \rightarrow R^{r} \stackrel{\psi}{\rightarrow} R^{m} \stackrel{\phi}{\rightarrow} R^{n} \rightarrow E \rightarrow 0
$$

be a minimal resolution of $E$. Since $E$ satisfies ( $\left.\mathscr{F}_{2}\right)$, we have

$$
n=v(E) \leqslant \operatorname{dim} R+\operatorname{rank}(E)-2,
$$

that is

$$
n-r=l=\operatorname{rank}(\phi)=n-\operatorname{rank}(E) \leqslant \operatorname{dim} R-2 .
$$

Since $r \neq 0$, the ideal $I_{r}(\psi)$ is, by induction, m-primary. From [6], however, we have

$$
\operatorname{dim} R=\operatorname{ht}\left(I_{r}(\psi)\right) \leqslant m-r+1=l-1,
$$

which is a contradiction.

In the discussion of the next cases we shall bring in the $Z$-complex of $E$.

Let $E$ be a module of projective dimension 3:

$$
0 \rightarrow R^{r} \stackrel{\theta}{\rightarrow} R^{s} \stackrel{\psi}{\rightarrow} R^{m} \stackrel{\phi}{\rightarrow} R^{n} \rightarrow E \rightarrow 0 .
$$

As above we assume $S(E)$ is reflexive and $R$ has lowest possible dimension. Further, assume $R$ contains a field.

Again from $\left(\widetilde{F}_{2}\right)$ we have $\operatorname{rank}(\phi)=l \leqslant d-2$. Since $L=\operatorname{image}(\phi)$ has depth $d$ -2 , by the induction hypothesis and the main result of [7], we may, in fact, assume $l=d-2$. Consider the Koszul complex of the embedding $L \rightarrow R^{n}$ (cf. §4).

$$
0 \rightarrow \stackrel{t}{\wedge} L \rightarrow \stackrel{t-1}{\wedge} \Lambda \otimes \otimes \tilde{S}_{1} \rightarrow \cdots \rightarrow L \otimes \tilde{S}_{t-1} \rightarrow \tilde{S}_{t} \rightarrow \operatorname{Sym}_{t}(E) \rightarrow 0 .
$$

If each $\wedge^{i} L, 1 \leqslant i \leqslant t$, has depth exceeding $i,(*)$ agrees with the corresponding subcomplex of $Z(E)$, cf. 4.1. Let us estimate the depth of these exterior powers. The free complex $\bigcup_{i}$ of [20 and 34] has length $\lambda_{i}=\inf \{i+r, 2 i\}$. If $\lambda_{i}-2+i \leqslant d-2$, $e_{i}$ is a minimal free resolution of $\wedge^{i} L$, which is, besides, an $i$ th syzygy module.

Suppose there exists $t$ such that

$$
\lambda_{t}+t \leqslant d \leqslant \lambda_{t}+t+1 \text {. }
$$

(Note that the right-hand side may not be the same as $\lambda_{t+1}+(t+1)$; also, in this range the $\wedge^{i} L$ will have depth $\geqslant i$.)

Chasing depths in the complex $(*)$ - taking into account that $\operatorname{Sym}_{t}(E)$ is reflexive and thus a second syzygy module-it follows that depth $\wedge^{t} L \geqslant t+1$ if $d-\lambda_{t}=t$ and depth $\wedge^{t} L \geqslant t+2$ if $d-\lambda_{t}=t+1$, which contradicts the existence of $t$. 
Unfortunately this does not occur for many values of $d$ and $r$. The first unsettled case in $d=8, r=3$. Nevertheless we have

Proposition 7.3. Let $E$ be a module of projective dimension 3. If the third Betti number $\beta_{R}^{3}(E)=1$ or 2 , then $S(E)$ is not factorial.

Let us now outline a construction which may lead to a counterexample to 7.1. It involves the $Z$-complex more intimately.

Suppose $I$ is an ideal of the regular local ring $R$ with the following properties:

(i) $\mathrm{ht}(I)=2$;

(ii) $I$ is generated by a $d$-sequence;

(iii) the powers $I^{t}, t \geqslant 1$, are unmixed.

For the construction, consider the module $\operatorname{Ext}_{R}^{1}(I, R)$ : At each associated prime $\mathfrak{p}$ of $I, I_{\mathfrak{p}}$ is generated by a regular sequence of 2 elements from (i) and (ii); therefore we can find an extension

$$
\xi: 0 \rightarrow R \rightarrow E \rightarrow 0
$$

which generates $\operatorname{Ext}_{R}^{1}(I, R)$ at each such prime.

Proposition 7.4. $S(E)$ is factorial.

Proof. We show that each $\operatorname{Sym}_{t}(E)$ is reflexive. For $t=1$ we use the choice of $\xi$ and Serre's lemma: Localizing at a prime $\mathfrak{p}$ of height 2 it follows that $E_{\mathfrak{p}}$ is free. If $\operatorname{ht}(\mathfrak{p}) \geqslant 3$ the unmixedness of $I$ guarantees depth $E \geqslant 2$. Thus $E$ is a second syzygy module.

For higher $t$ consider the exact sequence induced by $\xi$ :

$$
0 \rightarrow \operatorname{Sym}_{t-1}(E) \otimes R \rightarrow \operatorname{Sym}_{t}(E) \rightarrow \operatorname{Sym}_{t}(I)=I^{t} \rightarrow 0,
$$

where (ii) is again used. From (iii) it follows that $\operatorname{Sym}_{t}(E)$ is reflexive along with $\operatorname{Sym}_{t-1}(E)$.

The conjecture asserts that if $R$ is regular then such ideals are perfect. Note that the complex $Z(E)$ will, by 5.6, be acyclic. If $S(E)$ is Cohen-Macaulay, we have by 6.7, that pd $E \leqslant 1$.

Let us argue that the conditions of 7.4 are highly unlikely by considering how the properties of the complex $M(I ; R)$ [14] place constraints on the homological properties of an ideal $I$ generated by a $d$-sequence.

Proposition 7.5. Let $R$ be a Gorenstein ring and I an ideal of height 2 generated by a d-sequence.

(a) If $I$ is unmixed, then $\mathrm{pd} I \neq 2$.

(b) If $I$ and $I^{2}$ are unmixed and $I$ is perfect at primes of height 5 , then pd $I \neq 3$ as well.

(c) If the powers $I^{t}, t \geqslant 1$, are unmixed and I satisfies sliding depth, then I is a Cohen-Macaulay ideal.

Proof. For a presentation $0 \rightarrow L=Z_{1}(E) \rightarrow R^{n} \rightarrow I \rightarrow 0$, we shall prove $L$ is a Cohen-Macaulay module. 
Since $I$ is generated by a $d$-sequence, the subcomplexes of $M(I ; R)$,

$$
\begin{aligned}
0 & \rightarrow H_{t} \rightarrow H_{t-1} \otimes \tilde{S}_{1} \rightarrow \cdots \rightarrow H_{1} \otimes \tilde{S}_{t-1} \rightarrow H_{0} \otimes \tilde{S}_{t} \\
& \rightarrow \operatorname{Sym}_{t}\left(I / I^{2}\right)=I^{t} / I^{t+1} \rightarrow 0
\end{aligned}
$$

are exact. Here $H_{t}$ denotes the homology of the Koszul complex $\mathscr{K}(\mathbf{a} ; R)$ associated to the $n$ generators in the presentation above. (Note that in this case, cf. 3.3, $Z\left(I / I^{2}\right)=M(I ; R)$.)

As $I$ is unmixed and $\operatorname{dim} R=d \geqslant 4$, making $t=1$ we have that $H_{1}$ is a submodule of $(R / I)^{n}$, and thus depth $H_{1} \geqslant 1$ in case (a) and depth $H_{1} \geqslant 2$ in case (b) (we have now used the fact that $d \geqslant 6$ for (b)). Using the complex for higher $t$ 's, we similarly get depth $H_{t} \geqslant 1,2$ in the respective cases (a), (b).

We use these values to estimate the depths of the modules of cycles of $\mathscr{K}(\mathbf{a} ; R)$. Denote by $B_{i}$ and $Z_{i}$ the boundaries and cycles of $\mathcal{K}(\mathbf{a} ; R)$. From the sequences $0 \rightarrow B_{i} \rightarrow Z_{i} \rightarrow H_{i} \rightarrow 0$ and $0 \rightarrow Z_{i+1} \rightarrow K_{i+1} \rightarrow B_{i} \rightarrow 0$ we get: In case (a), depth $Z_{i} \geqslant 3$, and in case (b), depth $Z_{i} \geqslant 4$, for all $i$.

Recall now (cf. 4.3) that $Z_{n-2}=Z_{1}^{*}=L^{*}$. We thus have

$$
\text { depth } L+\operatorname{depth} L^{*} \geqslant d-\operatorname{pd} I+1+\{3(\mathrm{a}) \text { or } 4(\mathrm{~b})\} \geqslant d+2 \text {. }
$$

By the duality theorem of $[\mathbf{1 0}], L$ is a Cohen-Macaulay module.

To prove (c), note that the sliding depth condition implies

$$
\text { depth } Z_{1}+\operatorname{depth} Z_{n-2} \geqslant(d-n+2)+(d-n+(n-1)=d+1+(d-n) \text {, }
$$

and all that remains - to use the duality theorem-is to show $d>n$. For that, consider the algebra $\operatorname{gr}_{I}(R)$. Since depth $I^{t} / I^{t+1} \geqslant 1$, we may, by [3], find an element $x \in \mathrm{m}=$ maximal ideal of $R$, regular on $\operatorname{gr}_{I}(R)$. It follows that the analytic spread, $l(I)$, is at most $d-1$. But for ideals with $S\left(I / I^{2}\right)=\operatorname{gr}_{I}(R), v(I)=l(I)$.

REMARKs. (i) Note that for $R=$ regular, (c) is already taken care by the construction of 7.4 and the criterion 6.8.

(ii) The earliest dimension for which 7.1 may fail is $d=5$. By $\left(\mathscr{F}_{2}\right)$, for such a module $E$ we must have $v(E) \leqslant 5+\operatorname{rank}(E)-2$, so we are in a situation similar to Example 4.4, i.e. $\operatorname{rank}(L)=3$ : Therefore the complex $Z(E)$ is acyclic. If $S(E)$ is known to be Cohen-Macaulay, the question will again be settled by 6.8 .

(iii) There is a partial converse to the construction 7.4. Indeed, if $E$ is a module of rank 2 for which $Z(E)$ is acyclic and $S(E)$ is factorial, we would have: From 5.6 there exists a Bourbaki sequence $0 \rightarrow R \stackrel{\psi}{\rightarrow} E \rightarrow I \rightarrow 0$, where $I$ is generated by a proper sequence and is unmixed. Since $S(E)$ is factorial it is clear that the element $e=\psi(1)$ is prime, and therefore $S(I)$ is a domain. From [13] it follows that $I$ is generated by a $d$-sequence ( $R=$ local, infinite residue field). Furthermore, the exact sequence

$$
0 \rightarrow E \otimes R \rightarrow \operatorname{Sym}_{2}(E) \rightarrow \operatorname{Sym}_{2}(I)=I^{2} \rightarrow 0
$$

implies that, if depth $E \geqslant 3$, then $I^{2}$ will also be unmixed. This may also be the case for the higher powers of $I$. 


\section{REFERENCES}

1. L. Avramov, Complete intersections and symmetric algebras, J. Algebra 73 (1981), 248-263.

2. L. Avramov and J. Herzog, The Koszul algebra of a codimension 2 embedding, Math. Z. 175 (1980), 249-280.

3. M. Brodmann, Asymptotic stability of Ass $\left(M / I^{n} M\right)$, Proc. Amer. Math. Soc. 74 (1979), 16-18.

4. W. Bruns, Zur Konstruktion basischer Elemente, Math. Z. 172 (1980), 63-75.

5. L. Davis, Ideals of the principal class, $R$-sequences and a certain monoidal transformation, Pacific $\mathrm{J}$. Math. 20 (1967), 197-205.

6. J. Eagon and D. G. Northcott, Ideals defined by matrices and a certain complex associated with them, Proc. Roy. Soc. 269 (1962), 188-204.

7. E. G. Evans and P. Griffith, The syzygy problem, Ann. of Math. (2) 114 (1981), 323-333.

8. J. Fogarty, Truncated Hilbert functors, J. Reine Angew. Math. 234 (1969), 65-88.

9. R. M. Fossum, The divisor class group of a Krull domain, Springer-Verlag, Berlin and New York, 1973.

10. R. Hartshorne and A. Ogus, On the factoriality of local rings of small embedding codimension, Comm. Algebra 1 (1974), 415-437.

11. J. Herzog and E. Kunz, Der kanonische Modul eines Cohen-Macaulay Rings, Lecture Notes in Math., vol. 238, Springer-Verlag, Berlin and New York, 1971.

12. J. Herzog, A. Simis and W. V. Vasconcelos, Approximation complexes of blowing-up rings. I, J. Algebra 74 (1982), 466-493.

13. , Approximation complexes of blowing-up rings. II, J. Algebra 82 (1983), 53-83.

14. Koszul homology and blowing-up rings, Proc. Trento Commutative Algebra Conf., Lectures Notes in Pure and Applied Math., vol. 84, Dekker, New York, 1983, pp. 79-169.

15. C. Huneke, On the symmetric algebra of a module, J. Algebra 69 (1981), 113-119.

16. _ Linkage and the Koszul homology of ideals, Amer. J. Math. 104 (1982), 1043-1062.

17. $\ldots$ On the symmetric and Rees algebras of an ideal generated by a d-sequence, J. Algebra 62 (1980), 268-275.

18. I. Kaplansky, Commutative rings, Univ. of Chicago Press, Chicago, 1974.

19. M. Kühl, On the symmetric algebra of an ideal, Manucripta Math. 37 (1982), 49-60.

20. K. Lebelt, Freie Auflösungen äußerer Potenzen, Manuscripta Math. 21 (1977), 341-355.

21. G. Levin and W. V. Vasconcelos, Homological dimensions and Macaulay rings, Pacific J. Math. 25 (1968), 315-324.

22. H. Matsumura, Commutative algebra, Benjamin-Cummings, New York, 1980.

23. M. Nagata, Local rings, Interscience, New York, 1962.

24. C. Peskine and L. Szpiro, Dimension projective finie et cohomologie lcoale, Inst. Hautes Étues Sci. Publ. Math. 42 (1973), 323-395.

25. E. Platte, Zur endlichen homologischen Dimension von Diffferentialmodul, Manuscripta Math. 32 (1980), 295-302.

26. D. Rees, On a problem of Zariski, Illinois J. Math. 2 (1958), 145-149.

27. M. E. Rossi, $A$ note on symmetric algebras which are Gorenstein rings, Comm. Algebra (to appear).

28. P. Samuel, Anneaux gradués factoriels et modules réflexifs, Bull. Soc. Math. France 92 (1964), 237-249.

29. A. Simis and W. V. Vasconcelos, The syzygies of the conormal module, Amer. J. Math. 103 (1981), 203-224.

30. _ On the dimension and integrality of symmetric algebras, Math. Z. 177 (1981), 341-358.

31. J. Tate, The arithmetic of elliptic curves, Invent. Math. 23 (1974), 179-206.

32. G. Valla, On the symmetric and Rees algebra of an ideal, Manuscripta Math. 30 (1980), 239-255.

33. U. Vetter, Zu einem Satz von G. Trautmann über der Rang gewisser koharenter analytischer Moduln, Arch. Math. 24 (1973), 158-161.

34. J. Weyman, Resolutions of the exterior and symmetric powers of a module, J. Algebra 58 (1979), $333-341$.

\section{Fachbereich Mathematik, Universität Essen, D - 4300 Essen 1, West Germany}

Departamento de Matemática, Universidade Federal de Pernambuco, 50.000 Recife, PerNAMBUCO, BRAZIL

Department of Mathematics, Rutgers University, New Brunswick, New Jersey 08903 